\title{
Sequence-dependent sensitivity explains the accuracy of decisions when cues are separated with a gap
}

\author{
Maryam Tohidi-Moghaddam ${ }^{1,2} \cdot$ Sajjad Zabbah $^{2} \cdot$ Farzaneh Olianezhad $^{2,3} \cdot$ Reza Ebrahimpour $^{1,2}$ \\ Published online: 10 July 2019 \\ (C) The Psychonomic Society, Inc. 2019
}

\begin{abstract}
Most decisions require information gathering from a stimulus presented with different gaps. However, the neural mechanism underlying this integration is ambiguous. Recently, it has been claimed that humans can optimally integrate the information of two discrete pulses independent of the temporal gap between them. Interestingly, subjects' performance on such a task, with two discrete pulses, is superior to what a perfect accumulator can predict. Although numerous neuronal and descriptive models have been proposed to explain the mechanism of perceptual decision-making, none can explain human behavior on this two-pulse task. In order to investigate the mechanism of decision-making on the noted tasks, a set of modified drift-diffusion models based on different hypotheses were used. Model comparisons clarified that, in a sequence of information arriving at different times, the accumulated information of earlier evidence affects the process of information accumulation of later evidence. It was shown that the rate of information extraction depends on whether the pulse is the first or the second one. Moreover, our findings suggest that a drift diffusion model with a dynamic drift rate can also explain the stronger effect of the second pulse on decisions as shown by Kiani et al. (Journal of Neuroscience, 33 (42), 16483-16489, 2013).
\end{abstract}

Keywords Perceptual decision-making $\cdot$ Discrete pulses $\cdot$ Random-dot motion $\cdot$ Accuracy $\cdot$ Accumulation to bound model

Electronic supplementary material The online version of this article (https://doi.org/10.3758/s13414-019-01810-8) contains supplementary material, which is available to authorized users.

Reza Ebrahimpour

rebrahimpour@srttu.edu

Maryam Tohidi-Moghaddam

m.tohidi@ipm.ir

Sajjad Zabbah

s.zabbah@ipm.ir

Farzaneh Olianezhad

f.olianezhad@ipm.ir

1 Faculty of Computer Engineering, Shahid Rajaee Teacher Training University, P.O. Box: 16785-136, Tehran, Iran

2 School of Cognitive Sciences, Institute for Research in Fundamental Sciences (IPM), P.O. Box: 19395-5746, Tehran, Iran

3 Faculty of Electrical Engineering, Shahid Rajaee Teacher Training University, P.O. Box: 16785-163, Tehran, Iran

\section{Introduction}

Struggling with an extensive range of decisions with various degrees of complexity and type is an inseparable part of life, from voting for a competent president by integrating different types of discrete information over a period of time to simple immediate decisions such as picking up a pen. Recent studies on perceptual decisions in primates have investigated the neurobiological mechanisms underlying simple decisions such as a binary choice between two likely stimuli (Britten et al., 1996; Heekeren et al., 2004; Horwitz et al., 2004; Kiani et al., 2008; Latimer et al., 2015; Newsome et al., 1989; Roitman \& Shadlen, 2002; Schall, 2003). Neural responses in the lateral intra-parietal area (LIP) gradually increase to reach a specific level of firing rate when a monkey wants to make a decision about the motion direction in a stimulus with its eye movement (Cook \& Maunsell, 2002; Gold \& Shadlen, 2001, 2007; Hanks et al., 2014; Heitz \& Schall, 2012; Huk \& shadlen, 2005; Kiani \& Shadlen, 2009; Kiani et al., 2008; Philiastides \& Sajda, 2005). Therefore, it has been hypothesized that a variety of perceptual and mnemonic decisions in primates can be explained by a "bounded evidence accumulation" framework (Kira et al., 2015; Philiastides \& Sajda, 2005; Ratcliff \& McKoon, 2008; Ratcliff \& Tuerlinckx, 
2002; Usher \& McClelland, 2001). A well-known class of models, drift diffusion model (DDM) and its extensions, has been proposed to explain this process based on the integration of noisy evidence in favor of different alternatives to reach a decision bound (Gold \& Shadlen, 2007; Link, 1992; Ratcliff $\&$ McKoon, 2008). Different extensions of the DDM can interpret the reaction time, accuracy, confidence, speedaccuracy trade-off, and some other aspects of decisionmaking (Hanks et al., 2014; Heitz \& Schall, 2012; Kiani et al., 2014; Smith \& Vickers, 1988; Usher \& McClelland, 2001; Wang, 2002).

Indeed, the vast majority of decisions involve a complicated procedure that is the accumulation of evidence from separated cues over time. Along the way, the brain needs to evaluate and integrate various pieces of information arriving at different times (Kiani et al., 2013; Kira et al., 2015; Yang \& Shadlen, 2007). Recently, it has been demonstrated that the brain can optimally accumulate evidence from a set of noisy observations and attach more weight to the more decisive evidence (Kira et al., 2015; Yang \& Shadlen, 2007). It has also been shown that humans can gather information from two discrete cues separated by a gap of time, independent of the length of gaps. More importantly, Kiani et al. indicated that the most recent evidence shows far more influence on decisions (Kiani et al., 2013). However, it still remains unclear which mechanism can explain the process of decision-making in the presence of two pulses separated by time gaps.

The aim of this study was to investigate the mechanism of decision-making of a two-pulse experiment suggested by Kiani et al. (2013). A behavioral experiment including trials with one (single-pulse) and two (double-pulse) pulses of random-dot motion, separated with different interim periods (similar to Kiani et al., 2013) was implemented. Behavioral results can replicate what Kiani et al. (2013) had previously clarified. First, subjects' accuracy improved in double-pulse trials more than what had been expected from single-pulse trials, and accuracy was independent of the interim period between pulses. Moreover, the second pulse had a larger effect on subjects' performance.

Using a well-known decision-making model, DDM, the plausible mechanism underlying this behavior was explored (Gold \& Shadlen, 2007; Mazurek et al., 2003; Shadlen et al., 2006). The DDM contains three basic parameters consisting of a starting point, drift rate, and width of the interval between decision thresholds (Bogacz et al., 2006; Ratcliff, 1978; Ratcliff \& Rouder, 1998; Ratcliff \& Tuerlinckx, 2002; Ratcliff et al., 2016). According to the parameters of the model, three different hypotheses were examined. Results showed that the process of information accumulation differs across double-pulse and single-pulse trials. The accumulated information in the first pulse plays the role of a starting point for the accumulation process in the second pulse. More importantly, the rate of accumulation, not the bound, depends on the sequence of pulse presentation. Interestingly, the proposed model can well explain the sequence-dependent behavior in trials with unequal pulse strengths, even when the parameters are fitted using different sets of trials with equal pulse strength.

\section{Material and methods}

\section{Subjects}

Four human subjects, two females and two males aged 24-31 years, were tested in this experiment. Two subjects (one female and one male) were naïve to the purpose of the experiment, and all subjects had normal or corrected-to-normal vision. Subjects signed an informed consent form before participating in the experiment. The Ethics Committee of Iran University of Medical Sciences approved the experimental protocol.

Before the main experiment, subjects were extensively trained (Supplemental Material Methods and Fig. S1) to achieve a high level of accuracy and a predefined reaction time (Fig. S2 and Fig. S3). In the main experiment, each subject completed 11-15 blocks containing 300 trials.

\section{Stimulus}

Subjects were asked to identify the direction of motion stimuli consisting of three independent sets of dots displayed on consecutive video frames (dot density: $16.7 \mathrm{dots} / \mathrm{deg}^{2} / \mathrm{s}$ ). The size of dots was $2 \times 2$ pixels (side: $0.088^{\circ}$ ) (Roitman \& Shadlen, 2002; Shadlen \& Newsome, 2001). Each set of white dots was shown on a black background for one frame $(13.3 \mathrm{~ms})$ and was updated three frames later (after $40 \mathrm{~ms}$ ). Therefore, the positions of the dots in frames one, two, and three were respectively updated in frames four, five, and six, and so on. To update, each dot was either moved coherently along the motion direction (right or left) at the velocity of $6 \mathrm{deg} / \mathrm{s}$ or relocated randomly. Task difficulty was controlled through the coherence of motion, which is the probability that each dot will be moved toward the motion direction.

\section{Procedure}

All experiments were conducted in a dimly lit room. Subjects were seated in front of a 19-in. LG CRT display monitor (screen resolution: $800 \times 600$, refresh rate: $75 \mathrm{~Hz}$ ) at a distance of $57 \mathrm{~cm}$. An adjustable chin rest and a forehead bar were used to ensure the stability of subjects' heads. MATLAB's Psychtoolbox (Brainard \& Vision, 1997; Pelli, 1997) was employed to display the stimuli. The procedure of trials is described below. 
1) Fixation: A red fixation point with a $0.3^{\circ}$ diameter at the center of the screen was on during the trial to maintain subjects' attention. Each trial started with the appearance of the red fixation point. Then, subjects were asked to gaze at the fixation point and maintain their gaze within the trial. Upon pressing the space key, the second step would start.

2) Target on: Two red targets with a $0.5^{\circ}$ diameter were presented on the right and left sides of the fixation point at a distance of $10^{\circ}$ to cue subjects for possible direction of motion. After a random delay (200-500 ms, truncated exponential), the motion stimuli were presented.

3) Motion: The motion stimulus was displayed within a $5^{\circ}$ circular aperture at the center of the monitor. As mentioned before, two types of stimulus presentation, singlepulse and double-pulse trials, were utilized as below:

- Single-pulse: Motion coherence was randomly selected from these values: $0 \%, 3.2 \%, 6.4 \%, 12.8 \%, 25.6 \%$, and $51.2 \%$. After $120 \mathrm{~ms}$ of stimulus presentation, the next step was followed (Fig. 1A).

- Double-pulse: In each pulse, motion coherence was randomly selected from these values: $3.2 \%, 6.4 \%$, and $12.8 \%$. However, both pulses had the same net direction of motion, and subjects were informed. Two pulses were separated by a gap from these values: $0,120,360$, or 1,080 ms. In total, there were $9 \times 4$ types of trials. Each pulse was presented for $120 \mathrm{~ms}$ and, after the second pulse, the next step was followed (Fig. 1B).

4) Delay: As in the second step, two targets and a fixation point were shown for a random delay (400-1,000 ms, truncated exponential).

5) Go: After the random delay, the fixation point extinguished and subjects were instructed to report their decision within $1 \mathrm{~s}$ after the Go cue by pressing two specific keys for right and left choices. After that, an auditory feedback with two frequencies (low frequency for correct and high frequency for error) beeped for correct and error responses. More importantly, on trials with $0 \%$ motion coherence, the frequency of auditory feedback was randomly selected.

Subjects completed one or two blocks a day, each block including 300 trials. In total, $42(6+36)$ types of trials were randomly interleaved in each block. Subjects were asked to perform each trial as accurately as possible. The probability correct of each block was displayed at the end of the block as a feedback to the subjects.

\section{Data analysis}

To estimate the effect of parameters on the binary choices, different logistic regression models were applied. In the following regression models, Logit $[\mathrm{P}]$ indicates $\log (P / 1-P)$ and $\beta_{\mathrm{i}}$ denotes fitted coefficients.

To approximate the performance of single-pulse trials, the first regression model was fitted as below:

$\operatorname{Logit}\left[P_{\text {correct }}\right]=\beta_{0}+\beta_{1} C$

where $\mathrm{C}$ is the coherency level of single-pulse trials.

To evaluate whether the probability correct is affected by the interim period or not, the following regression analysis was utilized:

$$
\begin{aligned}
\operatorname{Logit}\left[P_{\text {correct }}\right]= & \beta_{0}+\beta_{1} C_{1}+\beta_{2} C_{2}+\beta_{3} T+\beta_{4} C_{1} T \\
& +\beta_{5} C_{2} T
\end{aligned}
$$

where $\mathrm{C}_{1}$ and $\mathrm{C}_{2}$ are the motion coherence of the first and second pulse, respectively, and $\mathrm{T}$ is the interim period. The null hypothesis is the lack of relationship between the probability correct and the interim period $\left(\mathrm{H}_{0}: \beta_{3-5}=0\right)$.

In the next step, a regression model was fitted to compare the expected accuracy of a perfect integrator to the observed performance on discrete trials:

$\operatorname{Logit}\left[P_{\text {correct }}\right]=\operatorname{Logit}\left[P_{e}\right]+\beta$

where $\mathrm{P}_{\mathrm{e}}$ is the expected probability correct resulting from a perfect accumulator (Eq. 4). A positive value for $\beta$ indicates that the observed accuracy is higher than the expected one.

$P_{e}=1-\Phi\left(0, e_{1}+e_{2}, \sqrt{2}\right)$

where $\Phi$ is the normal cumulative distribution function, and is calculated as:

$\Phi(s, \mu, \sigma)=\int_{-\infty}^{0} N(v, \quad \mu, \sigma) d v$

where $N(v, \mu, \sigma)$ is the normal probability density function with mean $(\mu)$ and $\operatorname{SD}(\sigma) . \mathrm{e}_{1}$ and $\mathrm{e}_{2}$ are the pieces of evidence of two pulses. The distribution of the evidence can be calculated from the probability correct of single-pulse trials:

$e_{i}=\Phi^{-1}\left(P_{i}, 0,1\right), \quad i=1,2$

where $\mathrm{P}_{\mathrm{i}}$ is the probability correct of single-pulse trials based on Eq. 1, and $\Phi^{-1}$ is the inverse of the normal cumulative distribution function in Eq. 5.

The following regression model was used to test the effect of pulse sequence on the probability correct:

$\operatorname{Logit}\left[P_{\text {correct }}\right]=\beta_{0}+\beta_{1}\left[C_{1}+C_{2}\right]+\beta_{2}\left[C_{1}-C_{2}\right]$

where $C_{1}$ and $C_{2}$ are the motion coherence of the first and second pulse, respectively, and $\beta_{2}$ represents the change in probability correct from the weak-strong pulse $\left(\mathrm{C}_{1}<\mathrm{C}_{2}\right)$ to the strong-weak one $\left(\mathrm{C}_{1}>\mathrm{C}_{2}\right)$. The null 


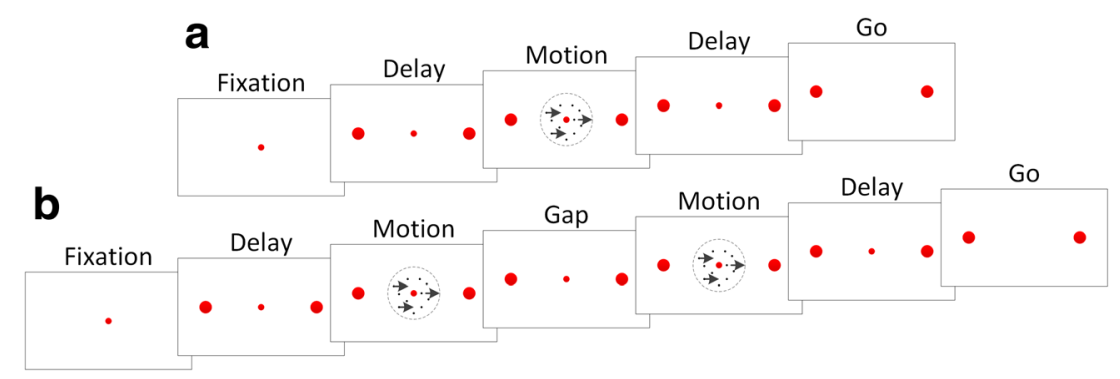

Fig. 1 Behavioral task paradigm. Subjects were asked to distinguish the direction of motion (left or right) in (a) single-pulse or (b) double-pulse trials. All trials started with a red fixation point in the center of the screen. Whenever the subject was ready and pressed a key, two red target points appeared on two sides of the fixation point for a random delay. Then,

hypothesis is that the probability correct does not depend on the pulse sequence $\left(\mathrm{H}_{0}: \beta_{2}=0\right)$.

\section{Modeling}

To explain the plausible mechanism for decisions with two discrete pulses, the DDM (Eq. 8) (Ratcliff, 1978; Ratcliff \& McKoon, 2008) implemented by Voss et al. was applied in a computationally efficient and accessible program called fastdm (Lerche \& Voss, 2017; Voss \& Voss, 2007; Voss et al., 2004, 2013).

$d v=V d t+\xi \sqrt{d t}$ where $v(0)=z$

This model is described via an accumulation-to-bound mechanism that starts with gathering noisy sensory evidence (explained by drift rate (v)) from a starting point (z) and terminates upon crossing the pre-specified response criterion (a). These three basic parameters existing in early versions of the diffusion model are compatible with both neurophysiological and behavioral data. The refined version of DDM was designated by seven parameters grouped into the following three classes: (I) the basic parameters of the decision process (decision threshold a, mean starting point $\mathrm{z}$, and mean drift rate $\mathrm{v}$ ), (II) the non-decision process parameter (non-decision time $t_{\mathrm{ND}}$ ), and (III) the changeability across-trial parameters (changeability in stimulus quality $\eta$, changeability in starting point sz, and starting point in non-decision time $\mathrm{st}_{\mathrm{ND}}$ ) (Ratcliff, 1978; Ratcliff \& McKoon, 2008; Ratcliff \& Tuerlinckx, 2002).

Fast-dm estimated the DDM's parameters using the partial differential equation (PDE) method (Voss \& Voss, 2007), which had swift calculations to compute the cumulative distribution function (CDF) and the Kolmogorov-Smirnov statistic (Voss et al., 2004). The diffusion model can describe the perceptual decision process in the brain and is actually a wellentrenched model in this field (Ratcliff, 1978; Ratcliff \& McKoon, 2008).

In this study and based on our hypothesis, three modified models were employed and compared using the Bayesian motion presentation (leftward or rightward random dot motion) started for one (a) or two (b) pulses. After stimulus presentation, fixation and two red target points were shown for a random delay. Finally, subjects received the Go cue and, after indicating their choice, they received feedback on their answers

information criterion (BIC) (Kass \& Wasserman, 1995; Liddle, 2007; Smith \& Spiegelhalter, 1980).

\section{Results}

Four human subjects were asked to make a decision about the net direction of motion in trials in which a 120 -ms motion pulse was displayed once or twice. Single-pulse and doublepulse trials were randomly presented. In double-pulse trials, both pulses had the same direction, and subjects were informed about this consistency. The net direction, motion strength, and interim period were random from trial to trial. Here, we first demonstrated that our data can reproduce the results reported by Kiani et al. (2013), and then fitted different DDMs to shed light on the mechanism of decision-making under discrete conditions.

Since $86 \%$ of trials were double-pulses, it may be hypothesized that the first pulse would be ignored and the subjects made their decisions merely based on the second pulse. However, the psychometric function of the single-pulse trials (Fig. 2) showed that the subjects accurately used the information of the first pulse. As expected from previous studies, the probability of being correct increased as a function of motion strength (Kiani et al., 2013), supporting the claim that subjects did not ignore the first pulse.

\section{Achieving the same accuracy levels independent of the interim period}

Consistent with previous results, accuracy was invariant in the interim period between pulses in double-pulse trials for both trials with equal pulse strength, or so-called same discrete trials (Fig. 3A, Eq. 2, $\beta_{3}=0.31 \pm 0.02, p=0.19 ; \beta_{4}=-0.05$ $\pm 0.03 \mathrm{p}=0.15$, for each subject; see Table S1) and trials with unequal pulse strength, or so-called different discrete trials (Fig. 3B, Eq. 2, $\beta_{3}=-0.11 \pm 0.29, p=0.70 ; \beta_{4}=0.01 \pm 0.02$ $\mathrm{p}=0.57 ; \beta_{5}=-0.002 \pm 0.02, \mathrm{p}=0.92$, for each subject; see Table S1) (see equivalence test result in Table 1). 


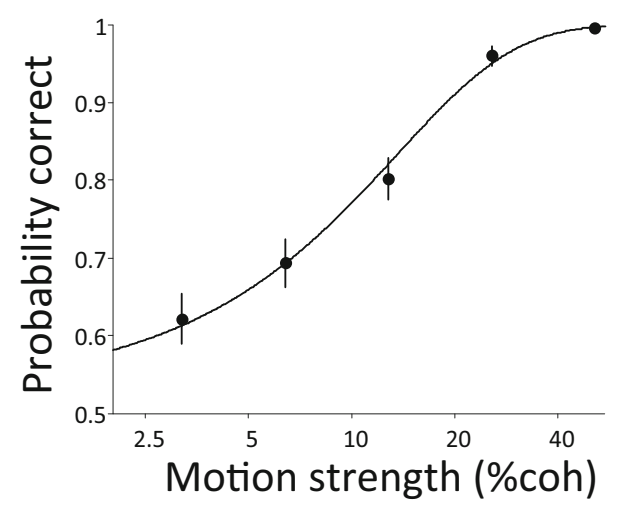

Fig. 2 Psychometric function of the single-pulse trials. Each data point represents pooled data across all single-pulse trials of all subjects. The solid curve is the fit of a regression model (Eq. 1) to the data. Error bars show the standard error of the mean

Accordingly, this finding demonstrated that the information of the first pulse was not lost but maintained throughout the interim period. Furthermore, the probability correct in weakstrong double-pulse trials was enhanced to above the expected performance of a perfect accumulator (Eq. 3, $\beta=0.10 \pm 0.04$, $\mathrm{p}=0.018$, for each subject; see Table $\mathrm{S} 2$ ), which, at least, provides evidence for the use of the information of both pulses. This better-than-anticipated performance also shows that the process of decision-making in double-pulse trials might be different from simply doubling the information of one pulse.

\section{What mechanism can explain the sequence-dependent performance of subjects in double-pulse trials?}

Our data also replicated what Kiani et al. (2013) had demonstrated about the sequence of pulses. As stated above, subjects performed better than expected in weak-strong sequence of double-pulse trials, where the second pulse is stronger than the first pulse and, as illustrated in Fig. 4, accuracy increased in double-pulse trials when the motion strength of the second pulse was stronger than that of the first one (Eq. 7, $\beta_{2}=0.03$ $\pm 0.005, \mathrm{p}=8.60 \times 10^{-8}$, for each subject; see Fig. S4).

In this part of the Results section, we propose a plausible mechanism for the observed sequence-dependent performance that caused the over-expected accuracy in double-pulse trials.

Assuming the accumulation to bound model as a true model of the decision process, we used the DDM to investigate three plausible hypotheses for the mechanism of the decision process in double-pulse trials: (1) Decision-making in doublepulse trials involves the same mechanism as single-pulse trials such that information accumulation in the first pulse will be continued in the second pulse until crossing a bound. This means that the rate of accumulation (drift rate) in the diffusion model depends on the summation of coherency of two pulses. (2) The first pulse affects the rate of information accumulation in the second pulse. (3) The first pulse affects the decision
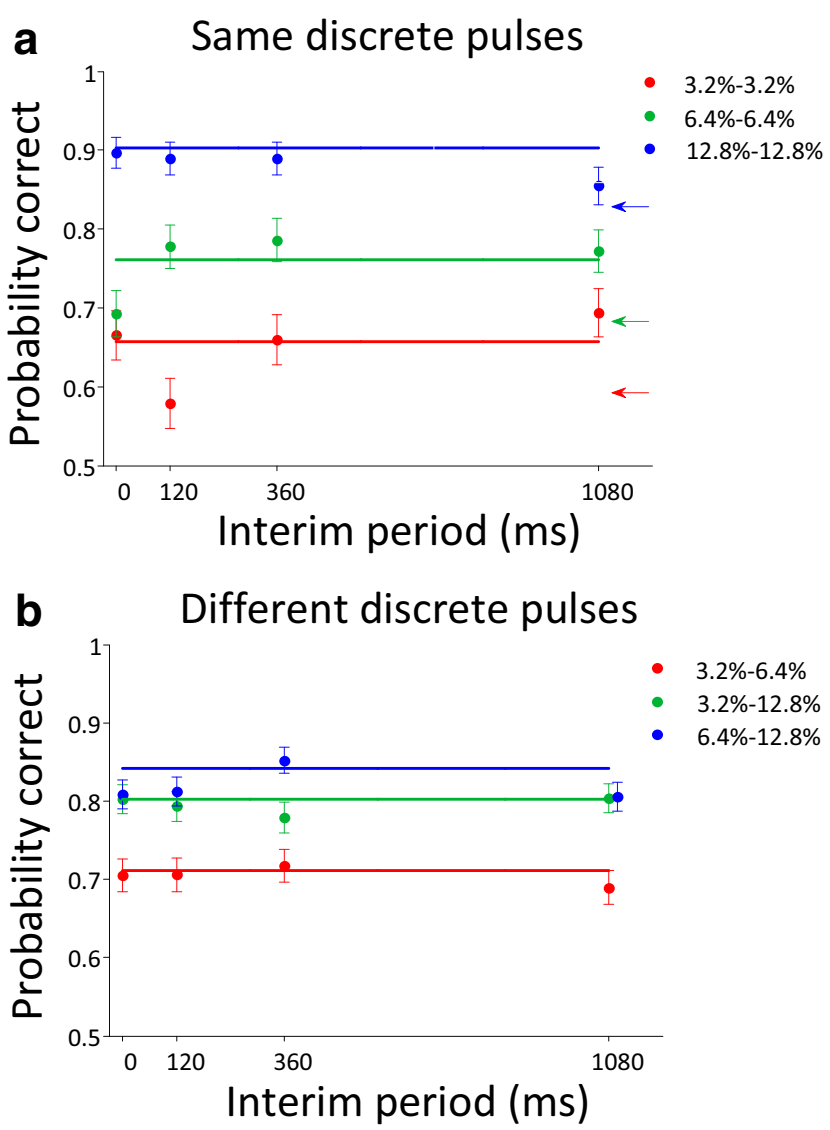

Fig. 3 Probability correct of double-pulse trials: (a) When the strength of both pulses was equal; arrows show the performance value derived from the fitted regression model (Eq. 1) to the relevant single-pulse trials; solid lines indicate the performance of the perfect accumulator (Eq. 3) according to the single-pulse accuracy. $\mathbf{b}$ When the strength of pulses was unequal; solid lines indicate the performance of the perfect accumulator (Eq. 3) according to the single-pulse accuracy; each data point shows pooled data from double-pulse trials with unequal pulses strength represented by legend and its reverse sequence (e.g., 3.2\%-12.8\%, 12.8\%, and $3.2 \%)$. Error bars show the standard error of the mean

threshold or the bound of information accumulation in the second pulse.

In the following section, the results of examining these three hypotheses are presented.

\section{Model fitting}

We used the DDM to test our hypotheses and examine the mechanism of decision-making in double-pulse trials. In so doing, based on our alternative hypotheses, we ran and compared three variations of the DDM to explore how and which parameters were influenced by discretizing information.

In the first model, we assumed that the information accumulation is similar in both single-pulse and double-pulse trials. This means that the interim period is not considerable, and the two pulses are considered as one pulse. To examine this assumption, we made the drift-rate $(v)$ a function of the sum of 
Table 1 Equivalence test results

\begin{tabular}{lllllll}
\hline Gap condition (ms) & 0 and 120 & 0 and 360 & 0 and 1,080 & 120 and 360 & 120 and 1,080 & 360 and 1,080 \\
\hline Motion strength & & & & & \\
$3.2-3.2 \%$ & $\mathrm{~B}= \pm 0.1029$ & $\mathrm{~B}= \pm 0.04$ & $\mathrm{~B}= \pm 0.0448$ & $\mathrm{~B}= \pm 0.1176$ & $\mathrm{~B}= \pm 0.1377$ \\
& $\mathrm{CI}=[0.07 ; 0.103]$ & $\mathrm{CI}=[-0.027 ; 0.036]$ & $\mathrm{CI}=[-0.043 ;-0.012]$ & $\mathrm{CI}=[0.048 ; 0.115]$ & $\mathrm{CI}=[-0.133 ;-0.095]$ & $\mathrm{CI}=[-0.065 ; 0.001]$ \\
& $\mathrm{P}=0.048$ & $\mathrm{P}=0.032$ & $\mathrm{P}=0.037$ & $\mathrm{P}=0.039$ & $\mathrm{P}=0.021$ & $\mathrm{P}=0.021$ \\
$6.4-6.4 \%$ & $\mathrm{~B}= \pm 0.1016$ & $\mathrm{~B}= \pm 0.1068$ & $\mathrm{~B}= \pm 0.1036$ & $\mathrm{~B}= \pm 0.0201$ & $\mathrm{~B}= \pm 0.021$ & $\mathrm{~B}= \pm 0.0309$ \\
& $\mathrm{CI}=[-0.099 ;-0.073]$ & $\mathrm{CI}=[-0.106 ;-0.081]$ & $\mathrm{CI}=[-0.098 ;-0.064]$ & $\mathrm{CI}=[-0.019 ; 0.002]$ & $\mathrm{CI}=[-0.011 ; 0.02]$ & $\mathrm{CI}=[-0.003 ; 0.028]$ \\
& $\mathrm{P}=0.022$ & $\mathrm{P}=0.044$ & $\mathrm{P}=0.016$ & $\mathrm{P}=0.03$ & $\mathrm{P}=0.044$ & $\mathrm{P}=0.027$ \\
$12.8-12.8 \%$ & $\mathrm{~B}= \pm 0.0217$ & $\mathrm{~B}= \pm 0.0232$ & $\mathrm{~B}= \pm 0.0597$ & $\mathrm{~B}= \pm 0.0145$ & $\mathrm{~B}= \pm 0.0472$ & $\mathrm{~B}= \pm 0.0461$ \\
& $\mathrm{CI}=[-0.005 ; 0.021]$ & $\mathrm{CI}=[-0.006 ; 0.022]$ & $\mathrm{CI}=[0.03 ; 0.056]$ & $\mathrm{CI}=[-0.011 ; 0.012]$ & $\mathrm{CI}=[0.025 ; 0.045]$ & $\mathrm{CI}=[0.023 ; 0.046]$ \\
$3.2-6.4 \%$ and & $\mathrm{P}=0.042$ & $\mathrm{P}=0.041$ & $\mathrm{P}=0.016$ & $\mathrm{P}=0.021$ & $\mathrm{P}=0.02$ & $\mathrm{P}=0.044$ \\
$6.4-3.2 \%$ & $\mathrm{~B}= \pm 0.0151$ & $\mathrm{~B}= \pm 0.0252$ & $\mathrm{~B}= \pm 0.0273$ & $\mathrm{~B}= \pm 0.0208$ & $\mathrm{~B}= \pm 0.0271$ & $\mathrm{~B}= \pm 0.0359$ \\
& $\mathrm{CI}=[-0.012 ; 0.01]$ & $\mathrm{CI}=[-0.022 ;-0.003]$ & $\mathrm{CI}=[0.005 ; 0.025]$ & $\mathrm{CI}=[-0.021 ;-0.002]$ & $\mathrm{CI}=[0.006 ; 0.026]$ & $\mathrm{CI}=[0.02 ; 0.036]$ \\
$3.2-12.8 \%$ and & $\mathrm{P}=0.017$ & $\mathrm{P}=0.012$ & $\mathrm{P}=0.026$ & $\mathrm{P}=0.049$ & $\mathrm{P}=0.037$ & $\mathrm{P}=0.048$ \\
$12.8-3.2 \%$ & $\mathrm{~B}= \pm 0.0187$ & $\mathrm{~B}= \pm 0.0327$ & $\mathrm{~B}= \pm 0.0111$ & $\mathrm{~B}= \pm 0.0225$ & $\mathrm{~B}= \pm 0.0175$ & $\mathrm{~B}= \pm 0.0345$ \\
& $\mathrm{CI}=[0.002 ; 0.016]$ & $\mathrm{CI}=[0.016 ; 0.032]$ & $\mathrm{CI}=[-0.009 ; 0.007]$ & $\mathrm{CI}=[0.008 ; 0.021]$ & $\mathrm{CI}=[-0.017 ;-0.004]$ & $\mathrm{CI}=[-0.032 ;-0.017]$ \\
$6.4-12.8 \%$ and & $\mathrm{P}=0.012$ & $\mathrm{P}= \pm 0.033$ & $\mathrm{P}=0.022$ & $\mathrm{P}=0.015$ & $\mathrm{P}=0.034$ & $\mathrm{P}=0.018$ \\
$12.8-6.4 \%$ & $\mathrm{CI}=[-0.011 ; 0.004]$ & $\mathrm{CI}=[-0.049 ;-0.037]$ & $\mathrm{CI}=[-0.003 ; 0.014]$ & $\mathrm{CI}=[-0.047 ;-0.031]$ & $\mathrm{CI}=[-0.001 ; 0.019]$ & $\mathrm{CI}=[0.039 ; 0.057]$ \\
& $\mathrm{P}=0.015$ & $\mathrm{P}=0.026$ & $\mathrm{P}=0.024$ & $\mathrm{P}=1$ & $\mathrm{P}=0.011$ & $\mathrm{P}=0.027$ \\
\hline
\end{tabular}

B, CI, and P represent equivalence bounds, 90\% confidence interval of TOST test, and p-value, respectively

the coherency of two pulses in double-pulse trials and called it model $_{\text {sum. }}$. The second and third models were fitted to explore whether the process of decision-making in the second pulse is influenced by the accumulated information of the first pulse or not. To do so, in the second hypothesis, we assumed that the accumulated information of the first pulse can affect the rate of information accumulation in the second pulse. In order to

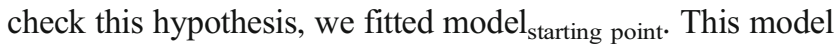

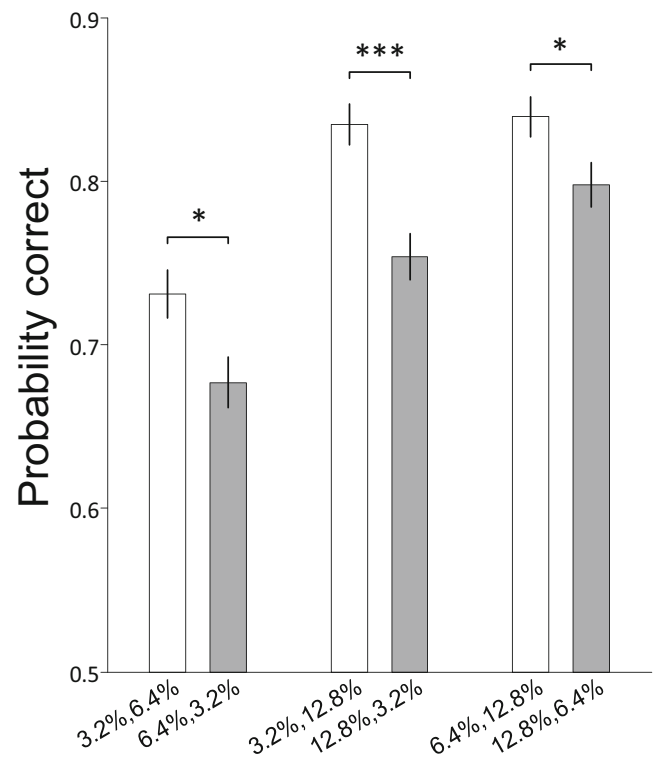

Fig. 4 Probability correct dependency on the sequence of motion pulses for double-pulse trials with unequal pulse strength. Probability correct was calculated by pooling data across all interim periods. Error bars show standard error of the mean. ${ }^{*} \mathrm{p}<0.05$ and ${ }^{* * * *} \mathrm{p}<1 \mathrm{E}-3$ is based on the assumption that in the second pulse the starting point of the accumulation process is equal to the accumulated evidence from the first pulse, which itself depends on the stimulus strength in that pulse. In other words, the starting point of the accumulation process in the second pulse depends on the drift rate in the first pulse. In model $l_{\text {starting point }}$, the starting point $(\mathrm{z})$ and the drift-rate $(\mathrm{v})$ of the DDM are a function of the motion strength of the first pulse and the second pulse, respectively. As a result, this model can potentially assign different drift rates for the first and the second pulses.

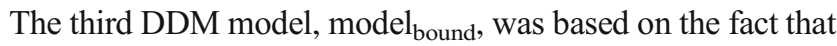
the decision bound determines the speed and accuracy tradeoff in which a higher decision bound results in higher accuracy with the cost of higher reaction time and a lower decision bound causes faster but less accurate decisions. Subjects may increase the decision bound when they receive weak information in the first pulse and expect a stronger one in the second

Table 2 Fitted parameters (mean $\pm \mathrm{SE}$ ) of the first DDM (model $\mathrm{sum}_{\text {sum }}$. $\mathrm{V}_{3.2-6.4}$ indicates the fitted drift-rate of pooled data from the pulse sequence and its reverse order (e.g., $3.2-6.4 \%$ and $6.4-3.2 \%$ )

\begin{tabular}{ll}
\hline$z$ & $0.5470 \pm 0.0159$ \\
$a$ & $1.0903 \pm 0.0170$ \\
$v_{3.2-6.4}$ & $0.7962 \pm 0.0956$ \\
$V_{3.2-12.8}$ & $1.2964 \pm 0.1159$ \\
$V_{6.4-12.8}$ & $1.3780 \pm 0.1045$ \\
$t_{N D}$ & $0.7101 \pm 0.0056$ \\
$s t_{N D}$ & $0.3382 \pm 0.0133$ \\
\hline
\end{tabular}


Table 3 Fitted parameters (mean \pm SE) of the second DDM (model starting $_{\text {. }}$ point)

\begin{tabular}{ll}
\hline$z_{-3.2}$ & $0.5206 \pm 0.0300$ \\
$z_{6} 6.4$ & $0.5425 \pm 0.0294$ \\
$z_{-12.8}$ & $0.5772 \pm 0.0290$ \\
$a$ & $1.0909 \pm 0.0238$ \\
$v_{3.2}$ & $0.7604 \pm 0.1348$ \\
$v_{6.4}$ & $1.0882 \pm 0.1430$ \\
$v_{12.8}$ & $1.6308 \pm 0.1674$ \\
$t_{N D}$ & $0.7181 \pm 0.116$ \\
$s t_{N D}$ & $0.3407 \pm 0.0150$ \\
\hline
\end{tabular}

pulse, and vice versa. Therefore, the third hypothesis is that the first pulse can influence the decision bound of the information accumulation in the second pulse (similar to Olianezhad et al., 2019). In order to test this hypothesis, we made the bound (a) of the DDM dependent on the motion strength of the first pulse and the drift-rate (v) on the motion strength of the second pulse. The fitted parameters of each model are listed in Tables 2, 3 and 4 (standard errors (SEs) were calculated with 100 iterations of bootstrap).

Here, we utilized the BIC (Kass \& Wasserman, 1995; Liddle, 2007; Smith \& Spiegelhalter, 1980) to compare the models (Table 5). As shown in this table, the BIC of model starting point is smaller than that of the other models, indicating that this model can better explain the observed behavior of subjects in double-pulse trials (see quantile probability function of the best model in Fig. 5).

In the next step, we also investigated the differences among the dependent parameters for the best model (model $_{\text {starting }}$ point). As mentioned in Table 3, the winner model produced three starting points and drift rates. Therefore, the significance of the differences among them was tested by the nonparametric bootstrap method (using a t-test with 100 iterations of bootstrap) (Efron, 1992; Efron \& Tibshirani, 1994). These differences were highly significant $\left(\mathrm{p}_{\mathrm{z} 3.2, \mathrm{z} 6.4}<0.05\right.$, $\mathrm{p}_{\mathrm{z} 3.2, \mathrm{z} 12.8}<0.05$, and $\left.\mathrm{p}_{\mathrm{z} 6.4, \mathrm{z} 12.8}<0.05\right)$, indicating that the winner model wins the competition not due to small differences between its unique free parameters. It also shows that the

Table 4 Fitted parameters (mean \pm SE) of the third DDM (model $l_{\text {boound }}$ )

\begin{tabular}{ll}
\hline$z$ & $0.5379 \pm 0.0222$ \\
$a_{-3.2}$ & $1.0829 \pm 0.0260$ \\
$a_{-6.4}$ & $0.0773 \pm 0.0216$ \\
$a_{-} 12.8$ & $1.1160 \pm 0.0238$ \\
$v_{3.2}$ & $0.8011 \pm 0.1162$ \\
$v_{6.4}$ & $1.1178 \pm 0.1270$ \\
$v_{12.8}$ & $1.6638 \pm 0.1452$ \\
$t_{N D}$ & $0.7131 \pm 0.0082$ \\
$s t_{N D}$ & $0.3419 \pm 0.0146$
\end{tabular}

Table 5 Model performance comparison via BIC and R2 metrics (mean \pm SD across subjects)

\begin{tabular}{llll}
\hline Model & Total parameters & $\mathrm{R}^{2}$ & BIC \\
\hline Model $_{\text {sum }}$ & 15 & $0.8181 \pm 0.0550$ & $-29.7407 \pm 2.8348$ \\
Model $_{\text {starting point }}$ & 14 & $0.8660 \pm 0.0573$ & $-35.1189 \pm 4.2153$ \\
Model $_{\text {bound }}$ & 14 & $0.7470 \pm 0.0884$ & $-29.0620 \pm 3.3791$ \\
\hline
\end{tabular}

accumulated evidence after the first pulse is significantly dependent on the pulse strength.

\section{Analyzing the parameters of the best model}

Table 3 illustrates the parameters of the best model. Considering that each pulse lasts $120 \mathrm{~ms}$, one can calculate the drift rate of the first pulse using Eq. 9:

$\mu=\frac{(z-0.5)}{0.120}$

where $\mu$ is the drift rate and $\mathrm{z}$ is the starting point for the second pulse or the accumulated information at the end of the first pulse. Moreover, 0.5 is the starting point of the first pulse. As a result, the drift rate of each stimulus at each pulse is presented in Table 6 .

Values in Table 6 suggest that the best model assigns larger values to the drift rate of the second pulse compared to the first pulse.

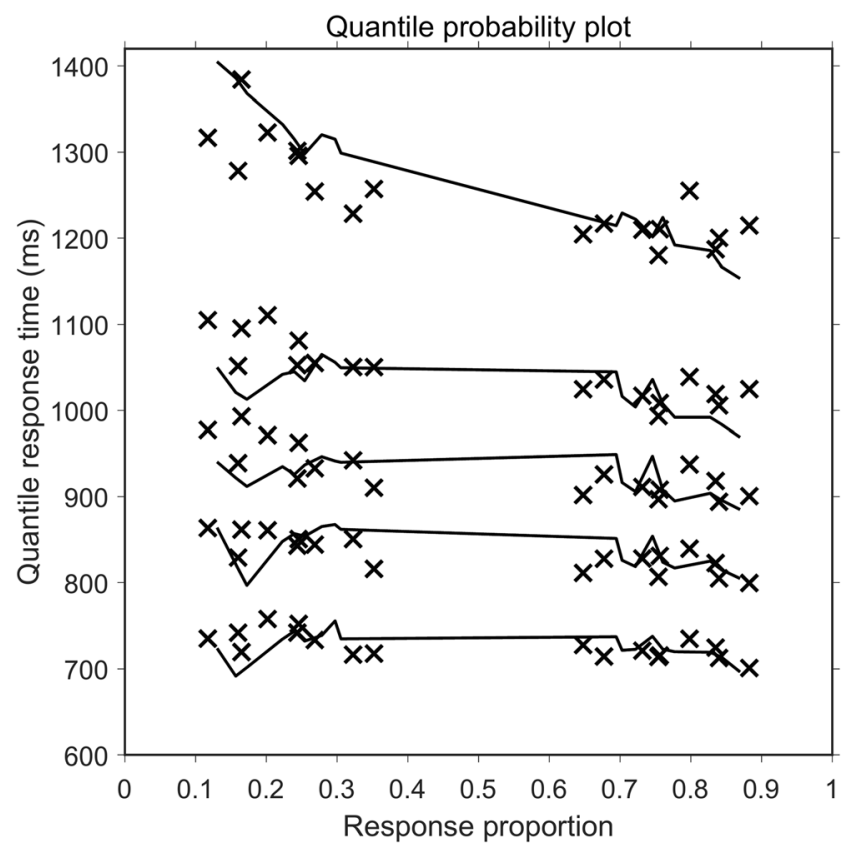

Fig. 5 Quantile-probability function for average data in the double-pulse trials. The percentage of correct responses is on the right side of the panel. In the left side we have the distributions for the error responses. The continuous lines show the fit of the Model $\mathrm{s}_{\text {starting point }}$ and the xs are the behavioral data. Response times are grouped to produce the five conditions 
Table 6 Drift rate of the first and second pulse

\begin{tabular}{lll}
\hline & First pulse & Second pulse \\
\hline$v_{3.2}$ & 0.172 & 0.6915 \\
$v_{6.4}$ & 0.3542 & 1.0125 \\
$v_{12.8}$ & 0.6433 & 1.5319 \\
\hline
\end{tabular}

\section{Model ${ }_{\text {starting point }}$ can explain the sequence-dependent behavior of subjects}

Figure $6 \mathrm{~A}$ demonstrates that model $l_{\text {starting point }}$ can mimic the same trend as behavioral data (Fig. 4). As reported by Kiani et al. (2013) and replicated here, subjects' accuracy in doublepulse trials is sequence-dependent. They rely more on the second pulse than on the first pulse to make their decisions. Here, we also investigate whether the best model suggested by BIC and fitted on double-pulse trials with equal pulse strength can replicate the sequence-dependent behavior of subjects in doublepulse trials with unequal pulse strength (Fig. 6B). It is noteworthy that, in Fig. 6B, the model is totally blind to the subject data in the double-pulse trials with unequal pulses strength.

\section{Discussion}

The decision-making process tends to integrate discrete pieces of information in favor of various options (Kiani et al., 2013; Kira et al., 2015; Yang \& Shadlen, 2007). Kiani et al. (2013) showed that, in a fixed-duration direction discrimination task, the integration of direction cues is independent of the temporal gap between them. Moreover, they revealed that participants' accuracy is more affected by the last pieces of information than the primary ones and, more importantly, their accuracy in double-pulse trials is more than what is expected from their performance in single-pulse trials. Here, using a similar psychophysics task, in addition to the confirmation of previous results, we suggested a mechanism to explain participants' behavior on such a task. Based on the DDM, we demonstrated that among a set of modified models, a sequence-dependent drift rate model can provide a better fit for the behavioral results. The model also accounts for both the sequence dependence and the over-expected accuracy of participants. It is notable that Kiani et al. (2013) concluded that the accuracy of weak-strong trials where the first pulse is weaker than the second one and strong-weak trials where the first pulse is stronger than the second one is significantly higher than that of the perfect integrator fitted to the performance of singlepulse trials. However, this over-expected accuracy is reproduced in our results only in trials with a weak-strong sequence. Our behavioral results, in contrast to Kiani et al. (2013), do not show a significant difference between the expected accuracy and the accuracy in trials with strong-weak sequence trials. As discussed by Kiani et al. (2013), "the better-than-expected performance in double pulse trials was actually explained by underperformance in single-pulse trials." Thus, performing better in singlepulse trials diminishes the better-than-expected performance effect. The discrepancy between our and Kiani's
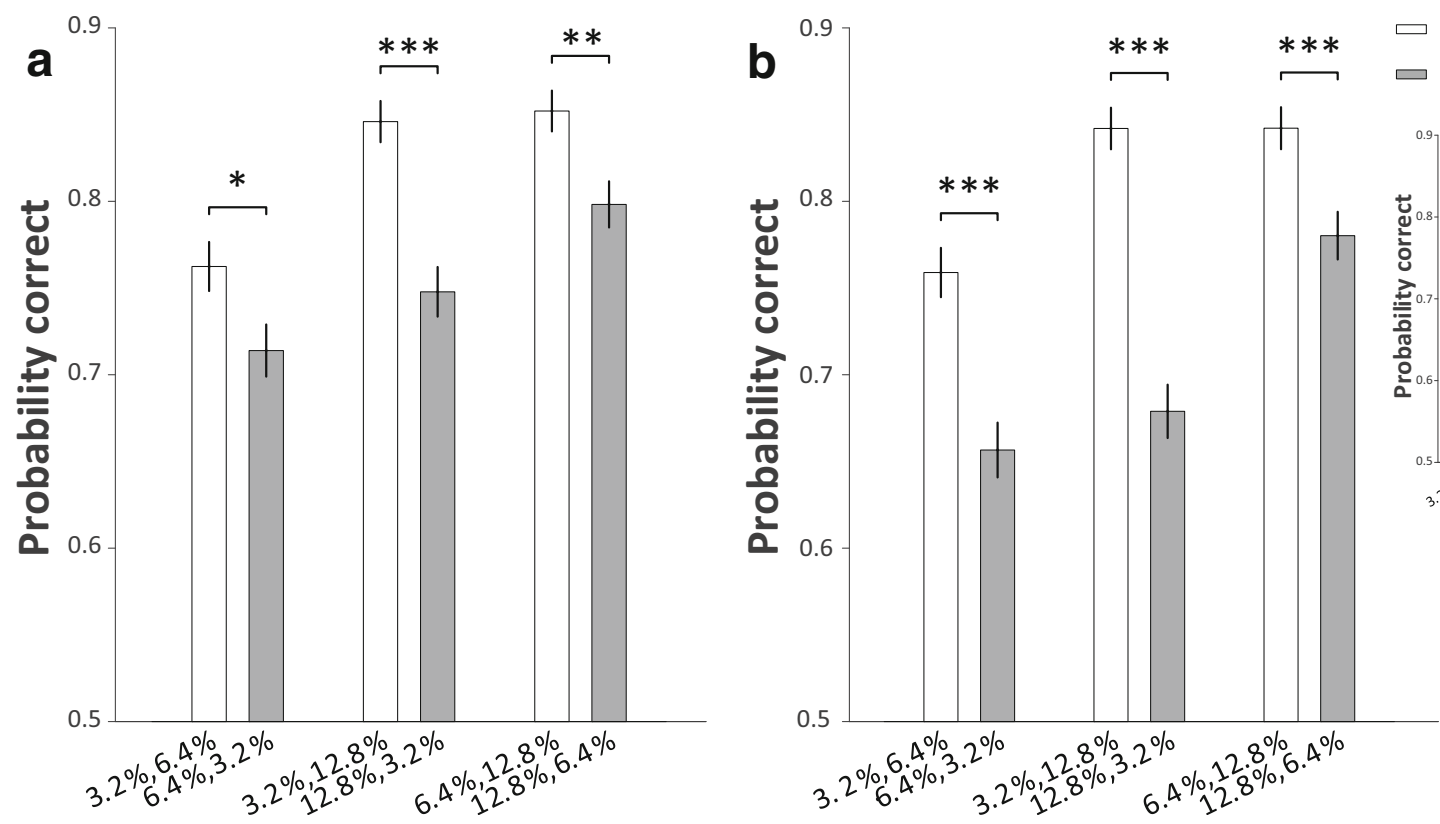

Fig. 6 (A) Simulated results of Fig. 4 with the fitted parameters of model $_{\text {starting point }}$ (B) Simulated results of Fig. 4 when we fitted the parameters of our model to double-pulse trials with equal pulse strength and simulated them for double-pulse trials with unequal pulse strength. The vertical bars of panel B show the results of the subjects (Fig. 4). Error bars denote standard error of the mean. ${ }^{* * *} \mathrm{p}<1 \mathrm{E}-3$. 
data stems from the fact that our subjects perform better than Kiani's subjects in single-pulse trials with low motion strength $(3.2 \%$ and $6.4 \%)$, which is evident in our psychometric functions.

Based on our results, the drift rate is not always a static parameter as assumed in previous studies. Based on Table 6, the rate of information extraction in the first pulse differs from that of the second pulse. This change in sensitivity also occurs in double-pulse trials with equal pulse strength in a way that the model fitted to double-pulse trials with equal pulse strength can truly predict the sequence-dependent performance in double-pulse trials with unequal pulse strength (Fig. 6B).

The change in the sensitivity of the second pulse can be explained by two potential mechanisms: (1) The time variant sensitivity (drift rate), according to which attention increases as the trial is near the end. This time variant decision-making has previously been suggested to explain longer reaction times in error trials (Ditterich, 2006). Ditterich showed that withintrial variability of sensitivity can better explain the distribution of reaction time in a random dot task than the variability in decision threshold. (2) The accumulated evidence (decision variable state) variant sensitivity (drift rate), according to which the sensitivity of the second pulse depends on the state of the accumulated evidence at the end of the first pulse. This hypothesis stems from the idea that when the accumulated evidence in the first pulse is less than what we expect, the brain will try to extract more information in the next pulse to improve his decision. On the other hand, when the accumulated evidence from the first pulse is more than what we expect, the brain does not need to increase the attention for gaining more evidence in the second pulse. As a result, the overall sensitivity improved in the second pulse. However, since both time and accumulated evidence are highly correlated variables, distinguishing between these hypotheses needs a more complex and wellcontrolled design of experiments.

In summary, our results shed more light on the mechanism of decision-making where pulses of information are separated by temporal gaps. We showed that the drift rate of the accumulation to bound model is modulated not only with the strength of the stimulus but also with the temporal position of pulses. However, our results investigated the process of decision-making when stimulus is discretized only in two pulses. More investigations into the process of decisionmaking with stimuli containing more than two pulses are needed to better understand the mechanism of decisionmaking in discretized environment.

Acknowledgements This work was supported by the Cognitive Sciences and Technologies Council under contract number 4916.

\section{References}

Bogacz, R., Brown, E., Moehlis, J., Holmes, P., \& Cohen, J. D. (2006). The physics of optimal decision making: a formal analysis of models of performance in two-alternative forced-choice tasks. Psychological review, 113(4), 700.

Brainard, D. H., \& Vision, S. (1997). The psychophysics toolbox. Spatial vision, 10, 433-436.

Britten, K. H., Newsome, W. T., Shadlen, M. N., Celebrini, S., \& Movshon, J. A. (1996). A relationship between behavioral choice and the visual responses of neurons in macaque MT. Visual neuroscience, 13(1), 87-100.

Cook, E. P., \& Maunsell, J. H. (2002). Dynamics of neuronal responses in macaque MT and VIP during motion detection. Nature neuroscience, 5(10), 985.

Ditterich, J. (2006). Evidence for time-variant decision making. European Journal of Neuroscience, 24(12), 3628-3641.

Efron, B. (1992). Bootstrap methods: another look at the jackknife. In Breakthroughs in statistics (pp. 569-593). Springer, New York, NY.

Efron, B., \& Tibshirani, R. J. (1994). An introduction to the bootstrap. CRC press.

Gold, J. I., \& Shadlen, M. N. (2001). Neural computations that underlie decisions about sensory stimuli. Trends in cognitive sciences, 5(1), $10-16$.

Gold, J. I., \& Shadlen, M. N. (2007). The neural basis of decision making. Annual review of neuroscience, 30.

Hanks, T., Kiani, R., \& Shadlen, M. N. (2014). A neural mechanism of speed-accuracy tradeoff in macaque area LIP. Elife, 3, e02260.

Heekeren, H. R., Marrett, S., Bandettini, P. A., \& Ungerleider, L. G. (2004). A general mechanism for perceptual decision-making in the human brain. Nature, 431(7010), 859.

Heitz, R. P., \& Schall, J. D. (2012). Neural mechanisms of speed-accuracy tradeoff. Neuron, 76(3), 616-628.

Horwitz, G. D., Batista, A. P., \& Newsome, W. T. (2004). Representation of an abstract perceptual decision in macaque superior colliculus. Journal of neurophysiology, 91(5), 2281-2296.

Huk, A. C., \& Shadlen, M. N. (2005). Neural activity in macaque parietal cortex reflects temporal integration of visual motion signals during perceptual decision making. Journal of Neuroscience, 25(45), 10420-10436.

Kass, R. E., \& Wasserman, L. (1995). A reference Bayesian test for nested hypotheses and its relationship to the Schwarz criterion. Journal of the american statistical association, 90(431), 928-934.

Kiani, R., \& Shadlen, M. N. (2009). Representation of confidence associated with a decision by neurons in the parietal cortex. Science, 324(5928), 759-764.

Kiani, R., Churchland, A. K., \& Shadlen, M. N. (2013). Integration of direction cues is invariant to the temporal gap between them. Journal of Neuroscience, 33(42), 16483-16489.

Kiani, R., Corthell, L., \& Shadlen, M. N. (2014). Choice certainty is informed by both evidence and decision time. Neuron, 84(6), 1329-1342.

Kiani, R., Hanks, T. D., \& Shadlen, M. N. (2008). Bounded integration in parietal cortex underlies decisions even when viewing duration is dictated by the environment. Journal of Neuroscience, 28(12), 3017-3029.

Kira, S., Yang, T., \& Shadlen, M. N. (2015). A neural implementation of Wald's sequential probability ratio test. Neuron, 85(4), 861-873.

Latimer, K. W., Yates, J. L., Meister, M. L., Huk, A. C., \& Pillow, J. W. (2015). Single-trial spike trains in parietal cortex reveal discrete steps during decision-making. Science, 349(6244), 184-187.

Lerche, V., \& Voss, A. (2017). Retest reliability of the parameters of the Ratcliff diffusion model. Psychological research, 81(3), 629-652. 
Liddle, A. R. (2007). Information criteria for astrophysical model selection. Monthly Notices of the Royal Astronomical Society: Letters, 377(1), L74-L78.

Link, S. W. (1992). The wave theory of difference and similarity. Psychology Press.

Mazurek, M. E., Roitman, J. D., Ditterich, J., \& Shadlen, M. N. (2003). A role for neural integrators in perceptual decision making. Cerebral cortex, 13(11), 1257-1269.

Newsome, W. T., Britten, K. H., \& Movshon, J. A. (1989). Neuronal correlates of a perceptual decision. Nature, 341(6237), 52.

Olianezhad, F., Zabbah, S., Tohidi-Moghaddam, M., \& Ebrahimpour, R. (2019). Residual Information of Previous Decision Affects Evidence Accumulation in Current Decision. Frontiers in behavioral neuroscience, 13, 9.

Pelli, D. G. (1997). The VideoToolbox software for visual psychophysics: Transforming numbers into movies. Spatial vision, 10(4), 437-442.

Philiastides, M. G., \& Sajda, P. (2005). Temporal characterization of the neural correlates of perceptual decision making in the human brain. Cerebral cortex, 16(4), 509-518.

Ratcliff, R. (1978). A theory of memory retrieval. Psychological review, $85(2), 59$.

Ratcliff, R., \& McKoon, G. (2008). The diffusion decision model: theory and data for two-choice decision tasks. Neural computation, 20(4), 873-922.

Ratcliff, R., \& Rouder, J. N. (1998). Modeling response times for twochoice decisions. Psychological Science, 9(5), 347-356.

Ratcliff, R., \& Tuerlinckx, F. (2002). Estimating parameters of the diffusion model: Approaches to dealing with contaminant reaction times and parameter variability. Psychonomic bulletin \& review, 9(3), 438481.

Ratcliff, R., Smith, P. L., Brown, S. D., \& McKoon, G. (2016). Diffusion decision model: current issues and history. Trends in cognitive sciences, 20(4), 260-281.

Roitman, J. D., \& Shadlen, M. N. (2002). Response of neurons in the lateral intraparietal area during a combined visual discrimination reaction time task. Journal of neuroscience, 22(21), 9475-9489.
Schall, J. D. (2003). Neural correlates of decision processes: neural and mental chronometry. Current opinion in neurobiology, 13(2), 182-186.

Shadlen, M. N., \& Newsome, W. T. (2001). Neural basis of a perceptual decision in the parietal cortex (area LIP) of the rhesus monkey. Journal of neurophysiology, 86(4), 1916-1936.

Shadlen, M. N., Hanks, T. D., Churchland, A. K., Kiani, R., \& Yang, T. (2006). The speed and accuracy of a simple perceptual decision: a mathematical primer. Bayesian brain: Probabilistic approaches to neural coding, 209-37.

Smith, A. F., \& Spiegelhalter, D. J. (1980). Bayes factors and choice criteria for linear models. Journal of the Royal Statistical Society. Series B (Methodological), 213-220.

Smith, P. L., \& Vickers, D. (1988). The accumulator model of two-choice discrimination. Journal of Mathematical Psychology, 32(2), 135-168.

Usher, M., \& McClelland, J. L. (2001). The time course of perceptual choice: the leaky, competing accumulator model. Psychological review, 108(3), 550.

Voss, A., \& Voss, J. (2007). Fast-dm: A free program for efficient diffusion model analysis. Behavior Research Methods, 39(4), 767-775.

Voss, A., Nagler, M., \& Lerche, V. (2013). Diffusion models in experimental psychology. Experimental psychology.

Voss, A., Rothermund, K., \& Voss, J. (2004). Interpreting the parameters of the diffusion model: An empirical validation. Memory \& Cognition, 32(7), 1206-1220.

Wang, X. J. (2002). Probabilistic decision making by slow reverberation in cortical circuits. Neuron, 36(5), 955-968.

Yang, T., \& Shadlen, M. N. (2007). Probabilistic reasoning by neurons. Nature, 447(7148), 1075.

Publisher's note Springer Nature remains neutral with regard to jurisdictional claims in published maps and institutional affiliations. 